\title{
Reduced Peripheral Blood Mononuclear Cell ROCK1 and ROCK2 Levels in Obstructive Sleep Apnea Syndrome
}

\author{
ŞENAY AYDIN ${ }^{1}$, CENGIZ ÖZDEMIR ${ }^{2}$, CEM İSMAIL KÜÇÜKALI ${ }^{3}$, \\ SINEM NEDIME SÖKÜCÜ${ }^{2}$, MURAT GIRIŞ ${ }^{3}$, UĞUR AKCAN ${ }^{3}$ and ERDEM TÜZÜN ${ }^{3}$ \\ ${ }^{1}$ Department of Neurology, Yedikule Chest Diseases and Chest Surgery \\ Training and Research Hospital, Istanbul, Turkey; \\ ${ }^{2}$ Department of Chest Diseases, Yedikule Chest Diseases and Chest Surgery \\ Training and Research Hospital, Istanbul, Turkey; \\ ${ }^{3}$ Department of Neuroscience, Aziz Sancar Institute of Experimental \\ Medical Research, Istanbul University, Istanbul, Turkey
}

\begin{abstract}
Background/Aim: Obstructive sleep apnea syndrome (OSAS) is associated with intermittent episodes of hypoxia, endothelial dysfunction and associated cardiovascular problems. Our aim was to investigate whether OSAS-related hypoxia alters the expression of rhoassociated protein kinase (ROCK), a marker of chronic hypoxia and endothelial dysfunction. Materials and Methods: ROCK1 and ROCK2 levels were measured by immunoblotting in peripheral blood mononuclear cells (PBMC) of 47 OSAS patients and 17 healthy controls. Results: OSAS patients showed significantly lower PBMC ROCK1 and ROCK2 levels than healthy controls in the morning, but not in the evening. ROCK1/2 levels were correlated with blood triglyceride, visceral adiposity index, minimum oxygen saturation, $C$-reactive protein concentration, lymphocyte levels and sleep efficiency. Conclusion: Intermittent hypoxia induced by OSAS does not permanently alter ROCK protein expression levels. OSAS appears to be associated with endothelial dysfunction through inflammation and lipid metabolism pathways.
\end{abstract}

Obstructive sleep apnea syndrome (OSAS) is one of the most frequent sleep disorders with a prevalence of $2-4 \%$ in the adult population, $3.1 \%-7.5 \%$ in men and $1.8 \%-5 \%$ in women

This article is freely accessible online.

Correspondence to: Erdem Tüzün, Department of Neuroscience, Institute for Experimental Medicine, Istanbul University, Istanbul, Turkey. Tel: +90 2124142000-33356, Fax: +90 2124142001, e-mail: drerdem@yahoo.com

Key Words: Obstructive sleep apnea syndrome, rho-associated protein kinase, ROCK, triglycerides, polysomnography, sleep.
(1-9). OSAS is characterized by repetitive episodes of apnea due to total compression of respiratory tract, intermittent hypoxia, reduced blood oxygen saturation and impaired sleep quality. It may cause difficulty sleeping, morning headache, daytime sleepiness, fatigue and reduced performance in daytime activities. Age, male gender and obesity have been listed among the risk factors of OSAS $(1-3,10)$. Moreover, OSAS has been associated with increased risk for arterial and pulmonary hypertension, cardiovascular and cerebrovascular disorders and metabolic syndrome (2, 11-14).

Although pathophysiological processes underlying OSAS have not been fully understood, inflammation, vascular endothelial dysfunction and altered body metabolism have been implicated $(15,16)$. Rho-associated protein kinase (ROCK) enzyme is known to be involved in regulation of vascular functions and cardiovascular disease pathogenesis. ROCK activation has been shown to enhance endothelial permeability and induce angiogenic processes through regulation of endothelial gene expression (17). In several studies, ROCK enzyme activity has been shown to be altered in patients characterized with cardiorespiratory problems that might potentially lead to chronic hypoxia of the target tissue (e.g. brain, heart etc.) $(18,19)$. In a single study, ROCK activity has been shown to be increased in OSAS in correlation with increased C-reactive protein (CRP) concentration and reduced flow-mediated dilatation in the brachial artery suggesting the presence of an interplay with ROCK and endothelial dysfunction (20).

The present study was conducted to find out whether intermittent hypoxia induced by OSAS might alter ROCK1 and ROCK2 expression in the peripheral blood and thus levels of these proteins can be used to extrapolate the intensity of hypoxia induced by OSAS. Clinical and laboratory features that might potentially be associated with ROCK expression levels were also investigated. 


\section{Materials and Methods}

Patients. A total of 47 consecutive male OSAS patients $(42.8 \pm 8.4$ years-old) and 17 age and gender matched healthy men $(39.2 \pm 8.7$ years-old) were prospectively recruited. To avoid the interference of sex hormones, no female patients or controls were recruited. Each participant underwent a detailed physical and neurological exam, blood pressure measurement, total blood count and blood biochemistry investigations. Blood biochemistry included acute phase reactants C-reactive protein (CRP) and ferritin. Participants with abnormal medical exam, blood pressure and laboratory results were not included. Moreover, individuals with a history of hypertension, diabetes mellitus, cardiovascular, cerebrovascular, liver or kidney disease and chronic inflammatory or autoimmune disorders were excluded. Height, weight, body mass index (BMI), body adiposity index (BAI) and visceral adiposity index (VAI) were recorded for each participant. The study was approved by the Institutional Review Board and each participant gave informed consent for the study.

Sleep recordings. A medical history regarding sleep disorders was taken in detail. Sleep status was investigated with evaluation of sleep habits, sleep symptoms and Epworth Sleepiness Scale (ESS). Full-night polysomnography (PSG) recordings (Embla TMN 7000 PSG Amplifier, Reykjavik, Iceland) were performed by two sleep specialists who were blinded for the clinical characteristics of the patients, as per previously published criteria (21). PSG included 16channels electroencephalography (placed according to the 10-20 international electrode placement system), right and left electrooculography, chin electromyography and electrocardiography. Thoracic and abdominal movements were recorded with piezoelectric strain gauges and body posture was evaluated with thoracic belt sensors. Respiration was recorded with oronasal thermocouples and nasal pressure transducer and continuous pulse oximetry monitorization was done. The following PSG parameters were evaluated: sleep efficiency index (total sleep time per time in bed), oxygen desaturation index (ODI), minimum and mean oxygen saturation, rapid eye movements (REM) and non-REM (NREM) apnea/hypopnea index (AHI), REM and NREM duration. Apnea was defined as at least $90 \%$ drop in airflow from baseline, which lasts at least $10 \mathrm{sec}$. Hypopnea was defined as at least $30 \%$ reduction in airflow that lasts at least $10 \mathrm{sec}$ and is associated with at least $4 \%$ decrease in arterial oxyhemoglobin saturation. AHI was defined as the total number of apneas and hypopneas per hour of sleep time. Apneas were classified as obstructive if respiratory efforts were present and central if respiratory efforts were absent during the event.

Immunoblotting. For measurement of peripheral blood mononuclear cell (PBMC) expression levels of ROCK1 and ROCK2, venous blood samples were obtained from each participant at 08.00 AM and 20.00 PM. PBMC were isolated by ficoll centrifugation and were stored at $-80^{\circ} \mathrm{C}$. PBMC were thawed, homogenized with cell lysis buffer $[20$ $\mathrm{mM}$ Tris $\mathrm{pH} 7.4,137 \mathrm{mM} \mathrm{NaCl}, 2 \mathrm{mM}$ ethylenediaminetetraacetic acid (EDTA) pH 7.4, 1\% Triton X-100, 25 mM $\beta$-glycerophosphate, $1 \mathrm{mM}$ phenylmethylsulfonyl fluoride (PMSF), $10 \mu \mathrm{g} / \mathrm{ml}$ aprotinin, 10 $\mu \mathrm{g} / \mathrm{ml}$ leupeptin, $10 \mu \mathrm{g} / \mathrm{ml}$ pepstatin and $100 \mathrm{mM}$ orthovanadate] and after 20 min of incubation on ice were subjected to centrifugation at $20,000 \times \mathrm{xg}$ at $4^{\circ} \mathrm{C}$ for $20 \mathrm{~min}$. Protein concentrations were determined by a BCA Protein Assay Kit (Pierce Biotechnology, Waltham, MA,
USA). Twenty $\mu \mathrm{g}$ of proteins were loaded and separated by $4-20 \%$ SDS-polyacrylamide gradient gel electrophoresis and then transferred to $0.45-\mu \mathrm{m}$ polyvinylidene fluoride membranes $(100 \mathrm{~V}, 80 \mathrm{~min})$. After blocking for $1 \mathrm{~h}$ in phosphate buffered saline with tween 20 (PBST) (10 $\mathrm{mM}$ sodium phosphate, $0.9 \% \mathrm{NaCl}$, and $0.1 \%$ Tween 20 ) containing 5\% non-fat dry milk, blots were incubated overnight at $4^{\circ} \mathrm{C}$ with the primary antibodies (1:500 dilution for all) to ROCK1, ROCK2 and $\beta$-actin (Abcam, Cambridge, UK) in PBST containing $3 \%$ non-fat milk. The blots were washed four times with PBST and incubated for 1 hour with horseradish peroxidase conjugated secondary antibody (1:2,000 dilution, Santa Cruz Biotechnology, Santa Cruz, CA, USA) in PBST. Immunoreactivity of the protein bands were detected by enhanced chemiluminescent autoradiography (ECL kit, Amersham Pharmacia Biotech, Piscataway, NJ, USA). A molecular weight standard (Bio-Rad Laboratories, Hercules, CA, USA) was loaded to assess relative molecular mass of detected bands. The immune blot bands were quantified through measurement of band intensity with ImageJ software using the same pixel scale for all pictures. Band intensities were normalized by $\beta$-actin expression and expressed as arbitrary units.

Statistical analysis. PBMC levels of ROCK1 and ROCK2 proteins were compared with ANOVA and Tukey's post-hoc test. Sleep and laboratory parameters of OSAS patients and healthy controls were compared with Student's $t$-test. Correlation studies were performed with Pearson's correlation test. $p<0.05$ was considered as statistically significant.

\section{Results}

Altered sleep and laboratory parameters of OSAS patients. Several parameters related with obesity, sleep dysfunction and inflammation were compared among OSAS patients and healthy controls (Table I). OSAS patients showed significantly higher weight, BMI and BAI values, whereas VAI values were comparable between OSAS patients and healthy controls. Among blood lipid levels, only total cholesterol was found to be significantly elevated in OSAS patients. As an acute phase reactant, blood CRP (but not ferritin) concentration was significantly increased in OSAS patients. Although OSAS patients showed trends towards exhibiting increased white blood cell, lymphocyte and neutrophil counts in the peripheral blood as compared to healthy controls, these differences did not attain statistical significance. Among sleep parameters, ODI, minimum oxygen saturation and REM/NREM apnea-hypopnea indices were significantly altered, whereas sleep efficiency, REM and NREM durations were not affected (Table I).

OSAS patients display reduced PBMC ROCK1/2 levels in the morning. Protein expression levels of ROCK1 and ROCK2 were measured and a semi-quantitative analysis of expression levels were done with a software. ROCK1 and ROCK2 levels of PBMC obtained in the morning were significantly lower than those obtained in the evening ( $p=0.003$ and $p=0.004$ for ROCK1 and ROCK2, 
Table I. Comparison of demographic, obesity, sleep, inflammation and blood lipid level parameters of obstructive sleep apnea syndrome (OSAS) patients and healthy controls. Values are indicated as mean \pm standard deviation.

\begin{tabular}{|c|c|c|c|}
\hline & OSAS patients $(n=47)$ & Healthy controls $(n=17)$ & $p$-Value \\
\hline Age & $42.8 \pm 8.4$ & $39.2 \pm 8.7$ & 0.134 \\
\hline Height (m) & $1.8 \pm 0.1$ & $1.8 \pm 0.1$ & 0.215 \\
\hline Weight $(\mathrm{kg})$ & $93.0 \pm 12.2$ & $80.1 \pm 10.3$ & $<0.001$ \\
\hline Body mass index $\left(\mathrm{kg} / \mathrm{m}^{2}\right)$ & $30.4 \pm 3.9$ & $25.7 \pm 2.8$ & $<0.001$ \\
\hline Body adiposity index (\%) & $27.9 \pm 3.1$ & $24.4 \pm 2.5$ & $<0.001$ \\
\hline Visceral adiposity index (AU) & $0.03 \pm 0.03$ & $0.03 \pm 0.03$ & 0.487 \\
\hline Triglyceride (mg/dl) & $234.7 \pm 209.1$ & $211.5 \pm 209.8$ & 0.350 \\
\hline Total cholesterol (mg/dl) & $219.5 \pm 46.0$ & $196.6 \pm 40.5$ & 0.032 \\
\hline HDL-cholesterol (mg/dl) & $42.4 \pm 8.3$ & $40.3 \pm 6.5$ & 0.153 \\
\hline LDL-cholesterol (mg/dl) & $129.5 \pm 38.4$ & $113.9 \pm 45.1$ & 0.109 \\
\hline C-reactive protein $(\mathrm{mg} / \mathrm{dl})$ & $4.2 \pm 3.8$ & $2.0 \pm 2.1$ & 0.003 \\
\hline Ferritin $(\mathrm{ng} / \mathrm{ml})$ & $60.8 \pm 31.8$ & $73.8 \pm 41.8$ & 0.127 \\
\hline White blood cell count (per $\mu \mathrm{l}$ ) & $7700 \pm 1400$ & $7000 \pm 1900$ & 0.079 \\
\hline Lymphocyte count (per $\mu l)$ & $2900 \pm 2100$ & $2300 \pm 700$ & 0.100 \\
\hline Neutrophil count (per $\mu \mathrm{l})$ & $4400 \pm 1200$ & $3900 \pm 1700$ & 0.176 \\
\hline Sleep efficiency $(\%)$ & $85.7 \pm 8.9$ & $85.5 \pm 8.9$ & 0.467 \\
\hline ODI (n/hour) & $26.7 \pm 20.8$ & $2.1 \pm 2.0$ & $<0.001$ \\
\hline Mean oxygen saturation (\%) & $96.7 \pm 30.2$ & $95.2 \pm 1.0$ & 0.368 \\
\hline Minimum oxygen saturation (\%) & $81.5 \pm 11.2$ & $91.0 \pm 2.6$ & $<0.001$ \\
\hline REM apnea index (n/hour) & $24.8 \pm 30.6$ & $4.0 \pm 5.5$ & $<0.001$ \\
\hline NREM apnea index (n/hour) & $130.3 \pm 121.2$ & $4.5 \pm 6.7$ & $<0.001$ \\
\hline REM hypopnea index (n/hour) & $11.4 \pm 13.0$ & $3.9 \pm 5.3$ & 0.001 \\
\hline NREM hypopnea index (n/hour) & $37.5 \pm 25.6$ & $3.1 \pm 3.8$ & $<0.001$ \\
\hline REM duration (minute) & $67.7 \pm 29.7$ & $70.6 \pm 22.8$ & 0.340 \\
\hline NREM duration (minute) & $326.3 \pm 39.0$ & $343.4 \pm 47.9$ & 0.100 \\
\hline
\end{tabular}

AU: Arbitrary units; n/hour: number/hour; ODI: oxygen desaturation index; REM: rapid eye movements; NREM: non-REM. Body mass index $=$ weight $(\mathrm{kg}) /$ height $(\mathrm{m})^{2}$. Body adiposity index $=\left[\right.$ hip circumference $(\mathrm{cm}) /$ height $\left.(\mathrm{m})^{1.5}\right]-18$. Visceral adiposity index $($ for men $)=[$ waist circumference $(\mathrm{cm}) / 39.68+(1.88 \times \mathrm{BMI})] \times[$ triglyceride level $(\mathrm{mmol} / \mathrm{l}) / 1.03] \times[1.31 / \mathrm{HDL}$-cholesterol level $(\mathrm{mmol} / \mathrm{l})]$.

respectively). Morning levels of both ROCK1 and ROCK2 were also significantly lower than morning and evening levels of healthy individuals ( $p<0.05$ for all comparisons) (Figure 1). There were no significant differences among OSAS patients and healthy controls by means of evening levels of ROCK1/2. Also, morning and evening levels of ROCK1/2 were identical in healthy individuals.

PBMC ROCK1/2 levels are correlated with triglyceride levels and visceral adiposity index. Potential associations between ROCK1/2 protein expression levels and measured lipid (triglyceride, total cholesterol, HDL-cholesterol, LDLcholesterol), inflammation (CRP, ferritin levels, white blood cell, lymphocyte, neutrophil counts), sleep (ODI, oxygen saturation, sleep efficiency, REM/NREM apnea-hypopnea indices, REM/NREM sleep duration) and obesity (weight, BMI, BAI, VAI) parameters were investigated by correlation studies. The most significant correlations $(p<0.001)$ were found between evening ROCK $1 / 2$ levels versus blood triglyceride levels and VAI values. By contrast, ROCK $1 / 2$ levels were not correlated with total cholesterol, HDLcholesterol, LDL-cholesterol, BMI and BAI values. Also, morning ROCK1/2 levels did not correlate with blood lipid levels and obesity index parameters. Significant correlations $(p=0.001)$ were also found between morning levels of ROCK1/2 versus CRP concentration and blood lymphocyte counts. Only evening ROCK1/2 levels of OSAS patients showed weak correlation $(p=0.016-0.029)$ with sleep efficiency, minimum oxygen saturation and non-REM sleep duration values (Table II). Representative scatter plot graphs of notable correlation studies for ROCK 1 expression levels are depicted in Figure 2. No significant correlation was found between ROCK1/2 levels and investigated laboratory and sleep parameters in healthy individuals (not shown).

\section{Discussion}

The Rho-ROCK pathway participates in various physiological processes including cell contraction, proliferation, differentiation, apoptosis and migration. Endothelial permeability and angiogenesis are also regulated by this pathway (22). Over the past decade, there has been increasing experimental evidence underlining the involvement of two isoforms of ROCK, ROCK1 and 

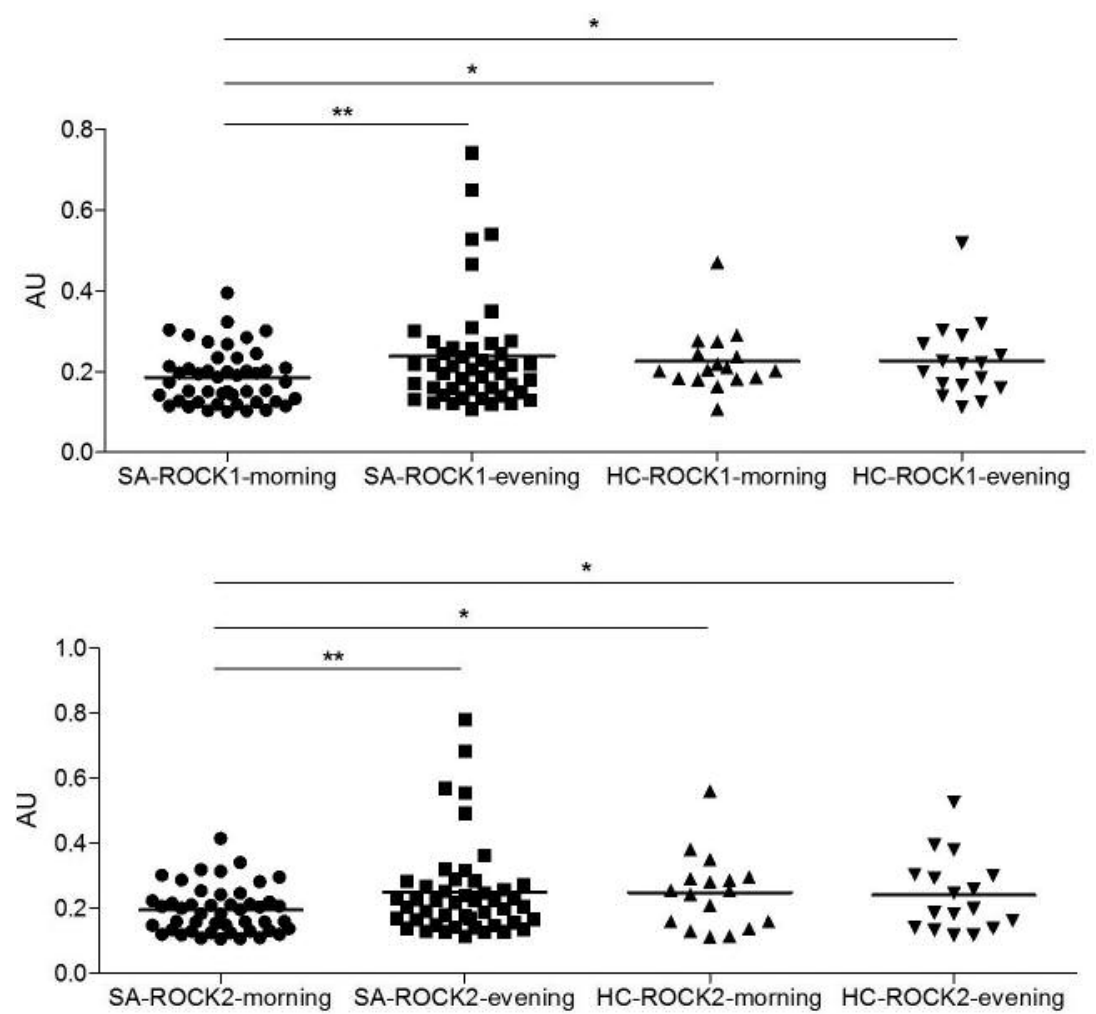

Figure 1. Peripheral blood mononuclear cell protein expression levels of rho-associated protein kinase (ROCK) 1 and ROCK2 in obstructive sleep apnea syndrome (SA) patients and healthy controls $(H C)$ as measured in the morning and evening. Protein expression levels were normalized with $\beta$-actin and expressed as arbitrary units (AU). Vertical lines indicate mean values. ${ }^{*} p<0.05 ;{ }^{* *} p<0.01$ as assessed by ANOVA and Tukey's post-hoc test.

Table II. List of notable correlations between obesity, sleep, inflammation and blood lipid level parameters and rho-associated protein kinase (ROCK) 1 and ROCK2 protein expression levels (as measured in the morning and evening by immunoblotting) in obstructive sleep apnea syndrome (OSAS) patients.

\begin{tabular}{|c|c|c|c|c|}
\hline p-Value; $R$ coefficient & $\begin{array}{l}\text { OSAS-ROCK1 } \\
\text { (Morning) }\end{array}$ & $\begin{array}{l}\text { OSAS-ROCK1 } \\
\text { (Evening) }\end{array}$ & $\begin{array}{l}\text { OSAS-ROCK2 } \\
\text { (Morning) }\end{array}$ & $\begin{array}{l}\text { OSAS-ROCK2 } \\
\text { (Evening) }\end{array}$ \\
\hline Triglyceride level (mg/dl) & $0.256 ; 0.169$ & $<0.001 ; 0.569$ & $0.254 ; 0.170$ & $<0.001 ; 0.543$ \\
\hline Visceral adiposity index (AU) & $0.261 ; 0.167$ & $<0.001 ; 0.529$ & $0.259 ; 0.168$ & $<0.001 ; 0.509$ \\
\hline C-reactive protein $(\mathrm{mg} / \mathrm{dl})$ & $0.001 ; 0.454$ & $0.273 ; 0.163$ & $0.001 ; 0.455$ & $0.275 ; 0.162$ \\
\hline Blood lymphocyte count & $0.001 ; 0.458$ & $0.868 ; 0.025$ & $0.001 ; 0.461$ & $0.870 ; 0.023$ \\
\hline Sleep efficiency $(\%)$ & $0.267 ;-0.165$ & $0.021 ;-0.338$ & $0.269 ;-0.165$ & $0.017 ;-0.348$ \\
\hline Minimum oxygen saturation (\%) & $0.409 ; 0.123$ & $0.020 ;-0.338$ & $0.414 ; 0.122$ & $0.016 ;-0.348$ \\
\hline Non-REM sleep duration (minute) & $0.363 ;-0.136$ & $0.029 ;-0.319$ & $0.368 ;-0.134$ & $0.029 ;-0.318$ \\
\hline
\end{tabular}

AU: Arbitrary units; REM: rapid eye movements. Significant correlations are denoted with bold characters. Values are indicated as p-value; R correlation coefficient of Pearson's correlation test.

ROCK2 in numerous cardiovascular and renal disorders. The activity of this pathway is increased in disorders such as heart failure, coronary artery disease and cerebrovascular disease, all of which associated with tissue hypoxia and reduced integrity of tissue vasculature $(18,19,23)$.
Particularly, ROCK2 is mostly expressed in endothelial cells and its expression is altered by several vascular disorders. Moreover, ROCK inhibitors have been shown to ameliorate pathophysiological and clinical adverse effects of these vascular disorders $(24,25)$. Recently, ROCK activity was 

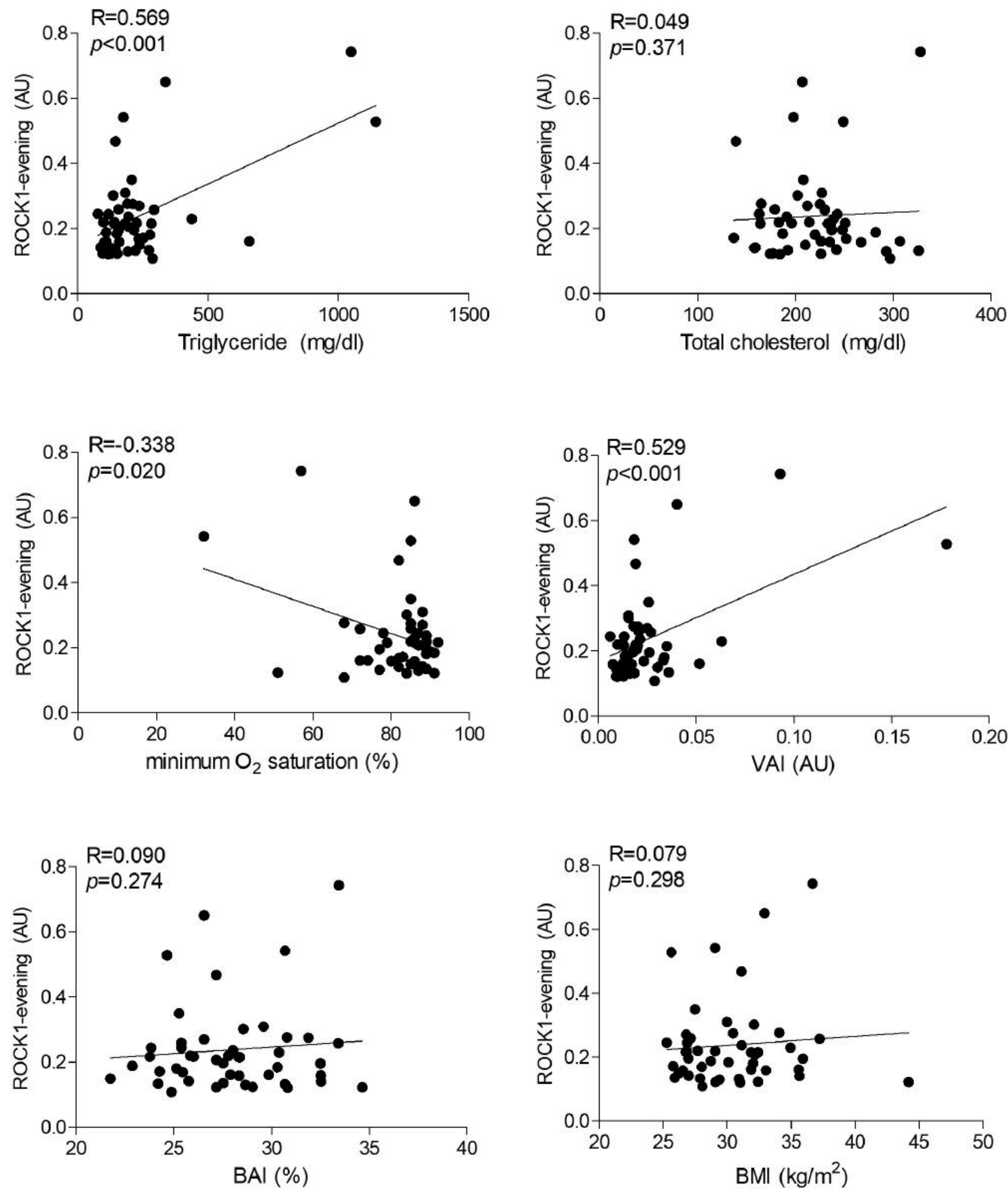

Figure 2. Representative scatter plot graphs of correlation studies between peripheral blood mononuclear cell rho-associated protein kinase (ROCK) 1 and ROCK2 protein expression levels in the evening versus blood lipid levels, minimum $\mathrm{O}_{2}$ saturation, visceral adiposity index (VAI), body adiposity index (BAI) and body mass index (BMI) values. Protein expression levels were normalized with $\beta$-actin and expressed as arbitrary units (AU). R: Correlation coefficient and p: p-value for Pearson's correlation test. 
found to be increased in OSAS suggesting that Rho-ROCK signaling pathway is also involved in this disease (20).

In this study, we investigated the expression levels of two ROCK isoforms in OSAS and found only a transient decrease in the expression levels of both isoforms in the morning, which was reversed in the evening. Moreover, ROCK1/2 levels were most significantly correlated with blood triglyceride concentration and VAI, which is a triglyceride-dependent factor. Moderate correlations were also determined between morning ROCK $1 / 2$ levels versus CRP levels and blood lymphocyte counts. In brief, most significant correlations were found with lipid metabolism and inflammation factors. Although ROCK1/2 expression levels were also found to be correlated with certain parameters of oxygen saturation and sleep functions, these correlations were rather weak and marginal. Overall, our results indicate that ROCK pathway is somewhat influenced by pathophysiological mechanisms of OSAS and suggest that endothelial dysfunction might be occurring in OSAS to a certain degree. However, this influence does not appear to be related with chronic intermittent hypoxia or cardiovascular alterations associated with OSAS but rather seems to be appearing as a function of lipid metabolism and inflammation pathway alterations.

The association between obesity and inflammation has since long been established and adipocytes are well known producers of proinflammatory cytokines. Breakdown of triglycerides has been shown to elicit proinflammatory responses in endothelial cells through activation of toll-like receptors and $\operatorname{NFk} \beta(26,27)$. Notably, in resemblance to our OSAS patients, in patients with metabolic syndrome, but without diabetes mellitus or hypertension, elevated levels of interleukin-6, soluble CD40L, monocyte chemoattractant protein and CRP have been found to be associated with altered ROCK activity (28). Moreover, both statins and specific ROCK pathway inhibitors such as fasudil reduce atherosclerosis risk through their parallel actions on lipid metabolism regulation and immunosuppression $(25,29)$. Thus, altered ROCK $1 / 2$ levels in OSAS patients might be the end result of endothelial dysfunction brought by metabolic syndrome and hypertriglyceridemia, which are frequently observed in OSAS patients, who have a higher prevalence of obesity than healthy controls.

An important finding in our study was the diurnal variation of ROCK $1 / 2$ levels, which was observed in OSAS patients, but not healthy controls. This disease-induced diurnal variation has been ignored in most previous studies assessing ROCK activity. Our results indicate that ROCK1/2 expression levels might start fluctuating under pathological conditions and this variation should be taken into account in ROCK pathway-based biomarker studies.

In conclusion, intermittent hypoxia induced by OSAS does not seem to be associated with ROCK $1 / 2$ expression changes. However, metabolic syndrome and obesity-induced inflammation might be the genuine culprits of endothelial dysfunction in OSAS leading to a transient decrease in ROCK $1 / 2$ levels. Whether this transient reduction is a compensating measure to counterbalance the hazardous effects of altered body metabolism in OSAS needs to be further investigated. ROCK $1 / 2$ expression levels might be used as potential biomarkers of metabolic syndrome in patients without clinically-noticeable body metabolism or cardiovascular disorders.

\section{Conflicts of Interest}

The Authors declare no conflicts of interest.

\section{References}

1 Young T, Palta M, Dempsey J, Skatrud J, Weber S and Badr S: The occurrence of sleep- disordered breathing among middleaged adults. N Engl J Med 328: 1230-1235, 1993.

2 Punchik B, Press Y and Goldbart A: Syndrome of obstructive sleep apnea in the elderly population-prevalence, clinical characteristics and treatment strategy. Harefuah 156: 41-44, 2017.

3 Duran J, Esnaola S, Rubio R and Iztueta A: Obstructive sleep apnea-hypopnea and related clinical features in a populationbased sample of subjects aged 30 to $70 \mathrm{yr}$. Am J Respir Crit Care Med 163: 685-689, 2001.

4 Bearpark H, Elliott L, Grunstein R, Cullen S, Schneider H, Althaus W and Sullivan C: Snoring and sleep apnea. A population study in Australian men. Am J Respir Crit Care Med 151: 1459-1465, 1995.

5 Ip MS, Lam B, Lauder IJ, Tsang KW, Chung KF, Mok YW and Lam WK: A community study of sleep-disordered breathing in middle-aged Chinese men in Hong Kong. Chest 119: 62-69, 2001.

6 Ip MS, Lam B, Tang LC, Lauder IJ, Ip TY and Lam WK: A community study of sleep-disordered breathing in middle-aged Chinese women in Hong Kong: prevalence and gender differences. Chest 125: 127-134, 2004.

7 Kim J, In K, Kim J, You S, Kang K, Shim J, Lee S, Lee J, Lee $\mathrm{S}$, Park $\mathrm{C}$ and Shin C: Prevalence of sleep-disordered breathing in middle-aged Korean men and women. Am J Respir Crit Care Med 170: 1108-1113, 2004.

8 Udwadia ZF, Doshi AV, Lonkar SG and Singh CI: Prevalence of sleep-disordered breathing and sleep apnea in middle-aged urban Indian men. Am J Respir Crit Care Med 169: 168-173, 2004.

9 Franklin KA and Lindberg E: Obstructive sleep apnea is a common disorder in the population-a review on the epidemiology of sleep apnea. J Thorac Dis 7: 1311-1322, 2015.

10 Stradling JR: Sleep-related breathing disorders. 1. Obstructive sleep apnoea: definitions, epidemiology, and natural history. Thorax 50: 683-689, 1995.

11 Javaheri S, Barbe F, Campos-Rodriguez F, Dempsey JA, Khayat R, Javaheri S, Malhotra A, Martinez-Garcia MA, Mehra R, Pack AI, Polotsky VY, Redline S and Somers VK: Sleep apnea: types, mechanisms, and clinical cardiovascular consequences. J Am Coll Cardiol 69: 841-858, 2017. 
12 Shawon MS, Perret JL, Senaratna CV, Lodge C, Hamilton GS and Dharmage SC: Current evidence on prevalence and clinical outcomes of co-morbid obstructive sleep apnea and chronic obstructive pulmonary disease: a systematic review. Sleep Med Rev 32: 58-68, 2017.

13 Mims Kn and Kirsch D: Sleep and stroke. Sleep Med Clin 11: 39-51, 2016.

14 Stansbury RC and Strollo PJ: Clinical manifestations of sleep apnea. J Thorac Dis 7: E298-310, 2015.

15 Kato M, Roberts-Thomson P, Phillips BG, Haynes WG, Winnicki $M$, Accurso $\mathrm{V}$ and Somers VK: Impairment of endothelium-dependent vasodilation of resistance vessels in patients with obstructive sleep apnea. Circulation 102: 26072610, 2000.

16 Wang J, Yu W, Gao M, Zhang F, Gu C, Yu Y and Wei Y: Impact of obstructive sleep apnea syndrome on endothelial function, arterial stiffening, and serum inflammatory markers: an updated meta-analysis and metaregression of 18 studies. J Am Heart Assoc 4: e002454, 2015.

17 Büssemaker E, Pistrosch F, Förster S, Herbrig K, Gross P, Passauer J and Brandes RP: Rho kinase contributes to basal vascular tone in humans: role of endothelium-derived nitric oxide. Am J Physiol Heart Circ Physiol 293: H541-547, 2007.

18 Bond LM, Sellers JR and McKerracher L: Rho kinase as a target for cerebral vascular disorders. Future Med Chem 7: 1039-1053, 2015.

19 Do e Z, Fukumoto Y, Sugimura K, Miura Y, Tatebe S, Yamamoto S, Aoki T, Nochioka K, Nergui S, Yaoita N, Satoh K, Kondo M, Nakano M, Wakayama Y, Fukuda K, Nihei T, Kikuchi Y, Takahashi J and Shimokawa H: Rho-kinase activation in patients with heart failure. Circ J 77: 2542-2550, 2013.

20 Zhang L, Zhuang JH, Peng H, Huang J, Huang LQ and Zhao ZX: Correlation between endothelial dysfunction, Rhoassociated protein kinase activity, C-reactive protein and obstructive sleep apnoea syndrome in male patients. J Int Med Res 40: 2183-2190, 2012.

21 Morgenthaler TI, Lee-Chiong T, Alessi C, Friedman L, Aurora RN, Boehlecke B, Brown T, Chesson AL Jr., Kapur V, Maganti R, Owens J, Pancer J, Swick TJ and Zak R: Standards of Practice Committee of the American Academy of Sleep Medicine Standards of Practice Committee of the American Academy of Sleep Medicine. Practice parameters for the clinical evaluation and treatment of circadian rhythm sleep disorders. An American Academy of Sleep Medicine report. Sleep 30: 14451459, 2007.
22 Komers R: Rho kinase inhibition in diabetic kidney disease. $\mathrm{Br}$ J Clin Pharmacol 76: 551-559, 2013.

23 Dong M, Liao JK, Yan B, Li R, Zhang M and Yu CM: A combination of increased Rho kinase activity and N-terminal pro-B-type natriuretic peptide predicts worse cardiovascular outcome in patients with acute coronary syndrome. Int J Cardiol 167: 2813-2819, 2013.

24 Shi $\mathrm{J}$ and Wei L: Rho kinases in cardiovascular physiology and pathophysiology: the effect of fasudil. J Cardiovasc Pharmacol 62: 341-354, 2013

25 Hao Z, Liu Y, Liao H, Zheng D, Xiao C and Li G: Atorvastatin plus metformin confer additive benefits on subjects with dyslipidemia and overweight/obese via reducing ROCK2 concentration. Exp Clin Endocrinol Diabetes 124: 246-250, 2016.

26 Welty FK: How do elevated triglycerides and low HDLcholesterol affect inflammation and atherothrombosis? Curr Cardiol Rep 15: 400, 2013.

27 Donath MY and Shoelson SE: Type 2 diabetes as an inflammatory disease. Nat Rev Immunol 11: 98-107, 2011.

28 Leguina-Ruzzi A, Pereira J, Pereira-Flores K, Valderas JP, Mezzano D, Velarde V and Sáez CG: Increased RhoA/RhoKinase activity and markers of endothelial dysfunction in young adult subjects with metabolic syndrome. Metab Syndr Relat Disord 13: 373-380, 2015.

29 Ma Z, Zhang J, Du R, Ji E and Chu L: Rho kinase inhibition by fasudil has anti-inflammatory effects in hypercholesterolemic rats. Biol Pharm Bull 34: 1684-1689, 2011.
Received December 11, 2017

Revised January 3, 2018

Accepted January 4, 2018 